\title{
Optical bifurcations in media with the Kerr nonlinearity
}

\author{
Krzysztof B. Zegadło* and Mirosław A. Karpierz \\ ${ }^{I}$ Faculty of Physics, Warsaw University of Technology, Koszykowa 75, 00-662 Warszawa
}

Received May 29, 2013; accepted June 27, 2013; published June 30, 2013

\begin{abstract}
A spontaneous symmetry breaking phenomenon or optical bifurcation occurs in media with some kind of symmetry when selffocusing nonlinearity is applied. In this manuscript that effect is investigated for the Kerr nonlinearity in structures with periodical distribution of a refractive index. Not only the symmetry bifurcation of the fundamental mode but also the properties of beam propagating in such structures have been studied. Spontaneous symmetry breaking has been presented both as a function of total beam power and as a function of relative refractive index change. The results can be utilized for all optical switching effects.
\end{abstract}

Spontaneous symmetry breaking or just bifurcation [1] is an effect which occurs in structures with symmetrical distribution of the refractive index where self-focusing nonlinearity is significant. Both wave propagation in a nonlinear medium and time evolution of Bose-Einstein condensate (BEC) is described by the same equation. It is the Nonlinear Schrödinger Equation (NLSE) [2] but in quantum mechanics it is also used as the Gross-Pitaevskii Equation [3]. When the total power of a beam (or energy of the Bose-Einstein condensate) exceeds some threshold value, the ground state symmetry is broken and the system minimizes energy in an asymmetrical state [4]. In a special case of an optical directional coupler, the distribution of the refractive index has two symmetrical maxima. Analogically, in Bose Einstein condensate such a special case is just the double well symmetrical potential. In these problems the ground state, which is the basic solution of the NLSE, is also symmetrical and its shape corresponds to the shape of the refractive index distribution or potential distribution for an optical structure and a double quantum well, respectively. Above the critical value of beam power (in the optical case) or the energy of condensate (in quantum mechanics case) only an asymmetrical solution of the NLSE is stable, and the light amplitude distribution is not the same for both cores of the coupler, similarly the distribution of cold atoms is not symmetrical in such a double well system. A spontaneous symmetry breaking effect has been observed in the BEC [5], as well as in nonlinear optics, e.g. in a photorefractive material [6]. Optical bifurcations have been studied in nonlinear optics in a directional coupler $[7,8]$, dual core fibers when a spatially continuous wave propagates [9], but also in dual core fibers for spatial solitons [10]. That phenomenon has also been observed

*E-mail: zegadlo@if.pw.edu.pl

for nonlinear coupled waveguides with the Bragg gratings [11] when the Kerr nonlinearity is applied. It has been studied in discrete systems, e.g. for solitons in dual core structures [12, 13]. For one-dimensional systems, e.g. one-dimensional lattice, the simplified model with nonlinearity concentrated only in cores and in a very close region around them can be used [14]. In that case, the discrete NLSE can be applied, where nonlinear terms are as delta functions.

In this paper the results in a structure with a double periodical distribution of the refractive index (Fig. 1b) are presented. Such a refractive index profile can be obtained by inserting a liquid crystal layer between a system of ITO electrodes [15] (Fig. 1a). All simulations have been run for a beam wavelength $\lambda=532 \mathrm{~nm}$.

a)
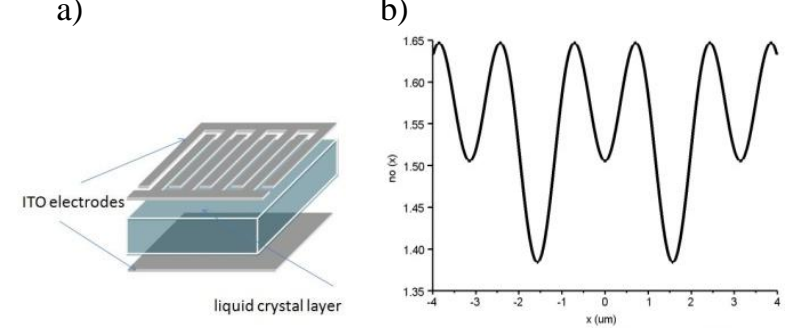

Fig. 1. a) Liquid crystal structure between two systems of ITO electrodes and b) corresponding effective refractive index profile $\mathrm{n}_{0}(\mathrm{x})$.

That refractive index distribution is a counterpart of potential in the BEC which has been analyzed both analytically and experimentally. For sufficiently deep minima in the refractive index distribution (like in the studied case), the beam launched to one of the two central cores will propagate only in these cores, switching, in a linear case, between them. It corresponds to a usual directional coupler which possesses only two cores, but light propagation is equivalent to that in the structure studied in this paper. Simulations of propagation properties have been carried out by using a standard Beam Propagation Method [16-23] with a scalar algorithm. This method calculates light amplitude by using values from the previous step. It can be modified by substituting an imaginary distance, the method being called ID-BPM [24-26]. The imaginary distance corresponds to an imaginary propagation constant which in such a form is an absorption coefficient. In 
consequence, all higher order modes (solutions of the NLSE) are absorbed and only the fundamental mode (with the highest propagation constant) survives. In the case of the Kerr nonlinearity, the refractive index is given as:

$$
\mathrm{n}(x)=\mathrm{n}_{0}(x)+n_{2}|\mathrm{E}(x)|^{2},
$$

where the first term is the linear refractive index distribution and the second is the nonlinear refractive index change $\Delta n$ depending on the local value of light intensity. Since the light amplitude is normalized, the total beam power is equal:

$$
\mathrm{P}=\int n_{2}|\mathrm{E}(x)|^{2} d x .
$$

It is convenient to divide the structure into two identical parts and calculate the power from $-\infty$ to $0\left(\mathrm{P}_{1}\right)$ and from 0 to $\infty\left(\mathrm{P}_{2}\right)$. Then the asymmetry coefficient can be defined as:

$$
\Theta=\frac{P_{1}-P_{2}}{P_{1}+P_{2}} .
$$

It can be applied to the description of the spontaneous symmetry breaking phenomenon, when the total power and the nonlinear refractive index change. The asymmetry of the basic NLSE solution (basic mode) has been presented as a function of total beam power (Fig. 2)

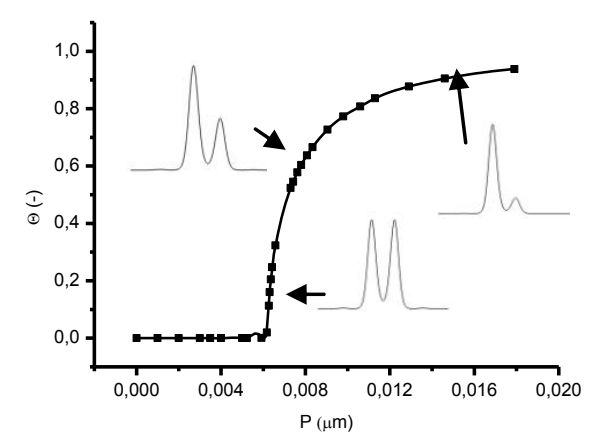

Fig. 2. Asymmetry coefficient of the beam intensity distribution (defined in Eq. (3)) as a function of total beam power.

This function breaks symmetry for power $\mathrm{P} \sim 0.006[\mu \mathrm{m}]$, but above this power value there still exists symmetrical mode, which is unstable and tends to an asymmetrical branch under the influence of disturbance. The asymmetry coefficient has also been investigated as a function of nonlinear relative refractive index change (Fig. 3).

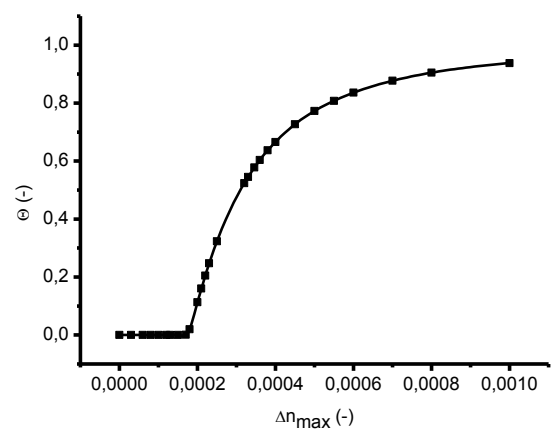

Fig. 3. Asymmetry coefficient of beam intensity distribution as a function of relative refractive index change $\Delta \mathrm{n}_{\max }$ in the maximal intensity of a beam.

In Fig. 2 one can see that there exists some threshold value of the power, above which the symmetry of fundamental mode is broken. The only solution which exists above this critical point is an asymmetrical solution. It tends with the power to all asymmetrical solutions, for which the asymmetry coefficient is 1 or -1 .

For numerical observations of beam propagating in the structure presented in Fig.1, the BPM method has been implemented. Propagation properties strongly depend on the way of beam launching. In a linear case, the beam launched to one core will switch to the other and back (Fig. 4).

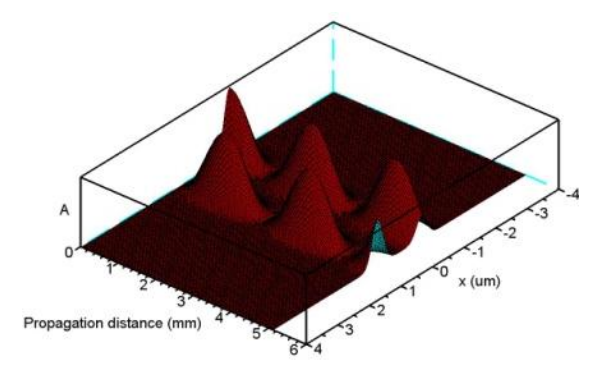

Fig. 4. Beam propagation in a linear case.

In this case, the beam oscillates between the left and right cores. It corresponds to the solution on the bifurcation diagram below the threshold power value when the basic mode is symmetrical. Such propagation properties are the result of superposition of two linear modes - basic solutions of the NLSE. The first is symmetrical and has two identical maxima and the second is antisymmetrical, which means in one core there is a maximum and in the other one - a minimum. The interference of these modes gives intensity distribution switching between the cores. In a nonlinear case, not all energy transfers to the neighbour core during propagation (Fig. 5). 


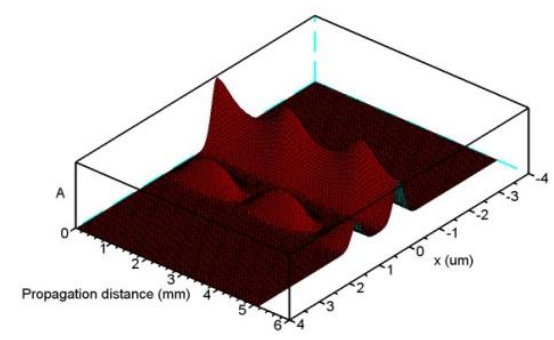

Fig. 5. Partially switching of the beam in a weak nonlinear regime.

Such propagation cannot be described analogically like in the previous case because it is nonlinear propagation which is not a superposition of nonlinear modes. The symmetrical solution in a strong nonlinear regime is unstable and transforms to the asymmetrical mode.

When nonlinearity is sufficiently strong there is no switching between both cores. The beam launched into one core will propagate only in that core and the asymmetry coefficient is then constant and equal to 1 or -1 (Fig. 6).

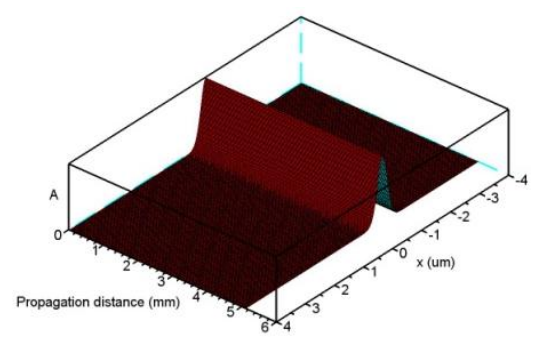

Fig. 6. Strong nonlinearity - no optical tunnelling effect.

In conclusion, below the critical point there exists a symmetrical solution and its counterpart - an antisymmetrical solution. Both are stable and the beam propagating in such a linear case is just a superposition of these two modes: all switches between the cores. Above the threshold power absolute value the asymmetry coefficient is higher than 0 , but lower than 1 , which means that a partially asymmetrical solution exists and during propagation not all power is tunnelling. The last case: $\theta=1$ or -1 and it corresponds to no switching in propagation and the only stable NLSE solution has the power in one of the cores.

The critical point, where symmetry is broken is neither phase transition of the first kind nor of the second. There is no discontinuity of $\theta$ function and its derivative is also continuous.

Further analytical and numerical investigations of the spontaneous symmetry breaking effect are both very prospective because, in that phenomenon, the beam power influences propagation properties. Light is used not only as a propagated signal but also as a steering signal. It allows to create optical systems where electrons are redundant, e.g. optical gates and other optical processing devices.

This work has been supported by the Marshal of the Mazovian Voievodeship in the frame of the European Social Funds in the Human Capital Operation Programme.

\section{References}

[1] D. Landau, E.M. Lifshitz, Quantum Mechanics (Moscow, Nauka 1974).

[2] Y.S. Kivshar, G.P. Agrawal, Optical Solitons: From Fibers to Photonic Crystals (New York, Academic 2003).

[3] S. Giorgini, L.P. Pitaevskii, S. Stringari, Rev. Mod. Phys. 80, 1215 (2008).

[4] E.A. Ostrovskaya, Y.S. Kivshar, M. Lisak, B. Hall, F. Cattani, D. Anderson, Phys. Rev. A 61, 031601 (2000).

[5] M. Albiez, R. Gati, J. Folling, S. Hunsmann, M. Cristiani, M.K. Oberthaler, Phys. Rev. Lett. 95, 010402 (2005).

[6] P.G. Kevrekidis, Z. Chen, B.A. Malomed, D.J. Frantzeskakis, M.I. Weinstein, Phys. Lett. A 340, 275 (2005).

[7] S.M. Jensen, IEEE J. Quantum Electron. QE-18, 1580 (1982).

[8] M.A. Karpierz, Opt. Commun. 90, 241 (1992).

[9] A.W. Snyder, D.J. Mitchell, L. Poladian, D.R. Rowland, Y. Chen, J. Opt. Soc. Am. B 8, 2102 (1991).

[10] C. Pare, M. Florjańczyk, Phys. Rev. A 41, 6287 (1990).

[11] W.C.K. Mak, B.A. Malomed, P.L. Chu, J. Opt. Soc. Am. B 15, 1685 (1998).

[12] G. Herring, P.G. Kevrekidis, B.A. Malomed, R. Carretero-Gonzalez, D.J. Frantzeskakis, Phys. Rev. E 76, 066606 (2007).

[13] Lj. Hadzievski, G. Gligoric, A. Maluckov, B.A. Malomed, Phys. Rev. A 82, 033806 (2010).

[14] V.A. Brazhnyi, B.A. Malomed, Phys. Rev. A 83, 053844 (2011).

[15] K.A. Brzdąkiewicz, M.A. Karpierz, A. Fratalocchi, G. Assanto, Optoelectron. Rev. 13, 107 (2005).

[16] J.E. Midwinter, Optical Fibers for transmission (London, John Wiley 1979).

[17] J. Van Roey, J. van der Donk, P.E. Lagasse, J. Opt. Soc. Am. 71, 803 (1981).

[18] Y. Chung, N. Dagli, J. Quantum. Electron. 26, 1335 (1990).

[19] D. Yevick, Opt. Quant. Electron. 26, 185 (1994).

[20] C. Ma, E. Van Keuren, Opt. Expr. 15, 402 (2007).

[21] F.A. Sala, M.A. Karpierz, Opt. Expr. 20, 13923 (2012).

[22] P.S. Jung, M.A. Karpierz, Opt. Commun. 285, 4184 (2012).

[23] P.S. Jung, M.A. Karpierz, Acta Phys. Polon. A 122, 829 (2012).

[24] S. Jungling, IEEE J. Quantum Elect. 30, 2098 (1994).

[25] C.L. Xu, W.P. Huang, S.K. Chaudhuri, J. Lightwave Technol. 11, 1209 (1993).

[26] Y.Z. He, F.G. Shi, Opt. Commun. 225, 151 (2003). 